\title{
Composition influences on the electrical and electromechanical properties of lead zirconate titanate thin films
}

P. Gerber, U. Böttger, and R. Waser

Citation: Journal of Applied Physics 100, 124105 (2006);

View online: https://doi.org/10.1063/1.2401047

View Table of Contents: http://aip.scitation.org/toc/jap/100/12

Published by the American Institute of Physics

\section{Articles you may be interested in}

Crystal orientation dependence of piezoelectric properties of lead zirconate titanate near the morphotropic phase boundary

Applied Physics Letters 72, 2421 (1998); 10.1063/1.121373

Ferroelectric thin films: Review of materials, properties, and applications

Journal of Applied Physics 100, 051606 (2006); 10.1063/1.2336999

Composition and orientation dependence of electrical properties of epitaxial $\mathrm{Pb}\left(\mathrm{Zr}_{x} \mathrm{Ti}_{1-\mathrm{x}}\right) O_{3}$ thin films grown using metalorganic chemical vapor deposition

Journal of Applied Physics 95, 3111 (2004); 10.1063/1.1645646

Thickness dependence of structural and electrical properties in epitaxial lead zirconate titanate films Journal of Applied Physics 86, 595 (1999); 10.1063/1.370772

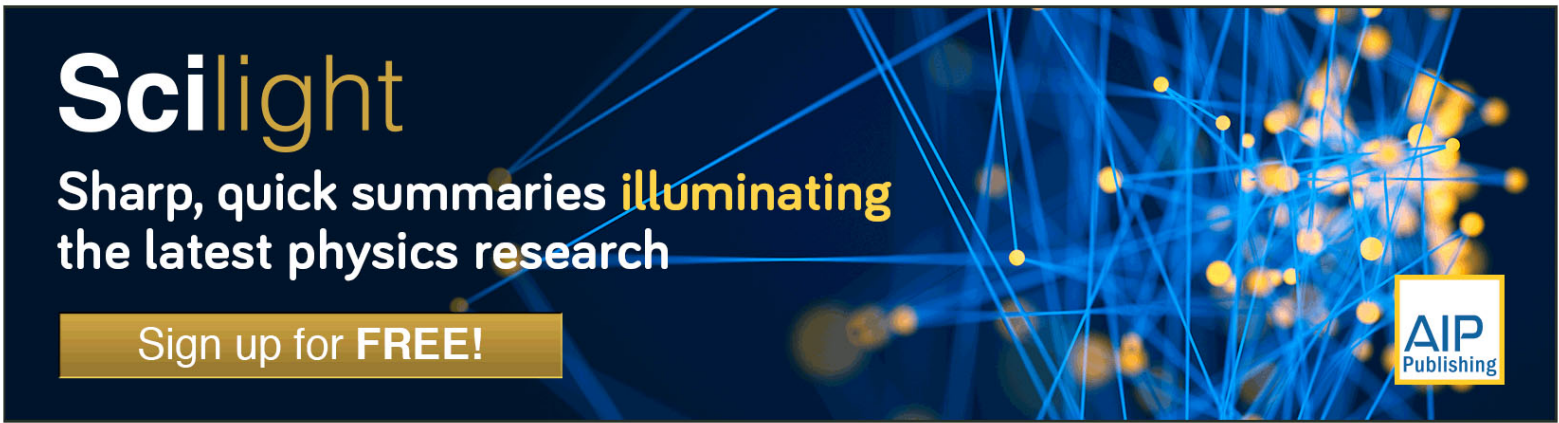




\title{
Composition influences on the electrical and electromechanical properties of lead zirconate titanate thin films
}

\author{
P. Gerber, ${ }^{\text {a) }}$ U. Böttger, and R. Waser \\ Institute of Materials in Electrical Engineering and Information Technology 2 (IWE2), Aachen University, \\ D-52074 Aachen, Germany
}

(Received 24 August 2006; accepted 30 September 2006; published online 21 December 2006)

\begin{abstract}
The influences of the $\mathrm{Zr}$ content on the structural, electrical, and electromechanical properties of $\mathrm{Pb}\left[\mathrm{Zr}_{(x)}, \mathrm{Ti}_{(1-x)}\right] \mathrm{O}_{3}[\mathrm{PZT}(x / 1-x)]$ thin films are investigated in detail. Additionally to measuring all major characteristics of the samples, the electromechanical large-signal behavior is modeled. Raising the $\mathrm{Zr}$ content increases the unit cell size and forces the preferred phase to become rhombohedral above the morphotropic phase boundary (MPB). The increased unit cell size changes the switching behavior and increases the intrinsic behavior of the unit cells. The intrinsic behavior is reduced by the phase change, which also introduces non $-180^{\circ}$ domain wall motion, improving the large-signal strain. Additionally, the domain configuration in saturation is more stable further away from the MPB. Finally, the most suitable materials will be selected for different applications.
\end{abstract}

(C) 2006 American Institute of Physics. [DOI: 10.1063/1.2401047]

\section{INTRODUCTION}

Ferroelectric $\mathrm{Pb}\left[\mathrm{Zr}_{(x)}, \mathrm{Ti}_{(1-x)}\right] \mathrm{O}_{3}[\mathrm{PZT}(x / 1-x)]$ ceramics exhibit superior ferroelectric, piezoelectric, and pyroelectric properties. Therefore, these materials are used in a wide range of applications such as actuators, force sensors, optical infrared sensors, and ferroelectric memories. In order to use these ceramics to their full potential, a thorough research of their properties is needed. ${ }^{1-3}$

One of the more interesting aspects in PZT thin film research is the influence of the material composition on the electrical and electromechanical properties. ${ }^{4-12}$ The major impact on the material properties results from the phase, in which the material changes upon cooling from the paraelectric phase. According to Ref. 13 thin films at room temperature show either the tetragonal or the rhombohedral phase for $\mathrm{Zr}$ ratios smaller or bigger than 53\%, respectively. Additionally, coexisting tetragonal and rhombohedral phases are found in epitaxial films near the morphotropic phase boundary. ${ }^{14,15}$ This results in an increased electromechanical behavior.

\section{APPLICATION REQUIREMENTS}

Depending on the application of the ferroelectric material, different characteristic properties are important for optimal suitability. In ferroelectric random access memory (FeRAM) applications, a high remanent polarization $P_{r}$ is one of the most important factors. A higher $P_{r}$ allows for a more secure information storage and a reduced electrode area of the device, especially with respect to down scaling. Depending on the device geometry and the voltage supply, the value of the optimal coercive voltage $V_{c}$ of the material can vary. However, higher coercive fields $E_{c}$ are usually accompanied with an increased $P_{r}$. If the material is used as dielectric in a capacitor, the polarization is not as important as the relative

${ }^{a)}$ Electronic mail: gerber@iwe.rwth-aachen.de permittivity. Again, higher values allow a reduction of the necessary electrode area. Electromechanical characteristics are less important, since they are not utilized in both applications. On the contrary, very high electromechanical strains might result in mechanical aging of the device, e.g., due to microcracks.

Concerning microelectro mechanical system (MEMS) devices, it is the other way around. Electrical properties are negligible or counterproductive, since high switching currents increase the power consumption of the device. Therefore, the polarization, especially the remanent $P_{r}$, is ideally a low value. With respect to the switching currents, it should be sufficient to search for materials with a low $P_{r} / P_{\text {sat }}$ ratio. This means that most of the polarization in saturation is lost if the field returns to zero, also known as backswitching. Additionally, the correlation, if any, between these high reversible extrinsic or intrinsic contributions to the polarization and the electromechanical properties $\left(S, d_{33}\right)$ is of interest. The latter should be as high as possible for any electromechanical application. Depending on the operating range, e.g., driving field, the most suitable material should be either selected by investigation of the piezoelectric small-signal coefficient $d_{33}$ or the field induced large-signal strain $S$. For instance, the ferroelectric material can be utilized in a sensor measuring mechanical energies, such as an applied force or acceleration, without application of an additional field (passive sensor). Hence, the working point is in and around the remanent state. Therefore, the material with the highest possible remanent $d_{33}$ is to be selected. Also, a high coercive field $E_{c}$ is advantageous to prevent the material from being driven out of its defined state by operation. On the other hand, piezoelectric actors or motors, which use most of their large-signal operating range, should be ideally driven by materials with the highest available strain at high fields.

In order to advice on material selections concerning the PZT composition, the electrical and electromechanical properties of several samples with increasing $\mathrm{Zr}$ content are mea- 
sured and compared. Prior to that, structural investigations are done to elucidate the reasons for any impacts on the material properties. Also, the electrical and electromechanical large-signal behavior is analyzed concerning the reversible and irreversible contributions. Finally, the best suitable material compositions for the given applications are selected using the results.

\section{SAMPLE PREPARATION AND PROPERTY MEASUREMENT}

The PZT $(45 / 55)$ thin films are prepared using chemical solution deposition (CSD) on double side polished $\mathrm{Pt}(111) / \mathrm{TiO}_{2} / \mathrm{SiO}_{2} / \mathrm{Si}$ substrates $(1 \mathrm{in} . \times 1$ in. $\times 0.5 \mathrm{~mm})$. After spin coating and pyrolysis of three coatings, the films are annealed in oxygen using rapid thermal annealing at $700^{\circ}$ for $5 \mathrm{~min}$. This results in a film thickness of $130 \mathrm{~nm}$. Pt top electrodes are sputter deposited with electrode areas ranging from 0.1 to $1 \mathrm{~mm}^{2}$. The back side is finally evaporation deposited with $\mathrm{Au}$ to achieve better reflectivity during electromechanical interferometric measurements.

Film orientation was determined by standard $\theta-2 \theta$ x-ray diffraction (XRD) und sample thickness is measured by a DEKTAK profilometer. The piezoelectric weak-signal coefficient $d_{33}$, large-signal strain $S$, and electrostriction coefficient $M_{33}$ are measured using a double-beam laser interferometer with a minimum resolution of $0.2 \mathrm{pm}$. For coefficient determination, the fast measurement method proposed in Ref. 16 is used. For this work, only electrodes with an area of $1 \mathrm{~mm}^{2}$ were used in order to minimize pad size influences. $^{17}$

The $\mathrm{Zr}$ contents of the investigated and compared samples are selected to reflect the materials used in various applications and also a broad spectrum of the material system. Compositions in the tetragonal regime, in this case PZT (30/70) and PZT (40/60), are known to be used in memory applications. ${ }^{18}$ PZT $(45 / 55)$ is the typical material for electromechanical applications ${ }^{19}$ due to its high piezoelectric response. Other material considered for this field are PZT (48/52) and PZT (53/47) since the compositions are next to and on the morphotropic phase boundary, respectively. Hence, there electromechanical properties should be high as well. Finally, as candidate for rhombohedral materials, PZT $(60 / 40)$ is investigated. $^{20}$

\section{RESULTS}

\section{A. Structural analysis}

Figure 1 depicts the structural analysis results of the samples. As can be seen in (a), all samples are mainly (111) oriented. To calculate the ratio of unit cells with different orientations, the count maximum for each phase is set into relation to the rest of the phase counts. The ratio of (111) oriented unit cells is as high as 90\%-96\% in tetragonal samples and drops to $70 \%$ for rhombohedral samples. Also, with higher $\mathrm{Zr}$ content the peaks are shifted towards lower beam angles, which indicates larger unit cells. The minor peak present in all samples is the (100) peak produced by unit cells with their axis of spontaneous polarization lying parallel to the sample surface and electrodes (in plane). Com-
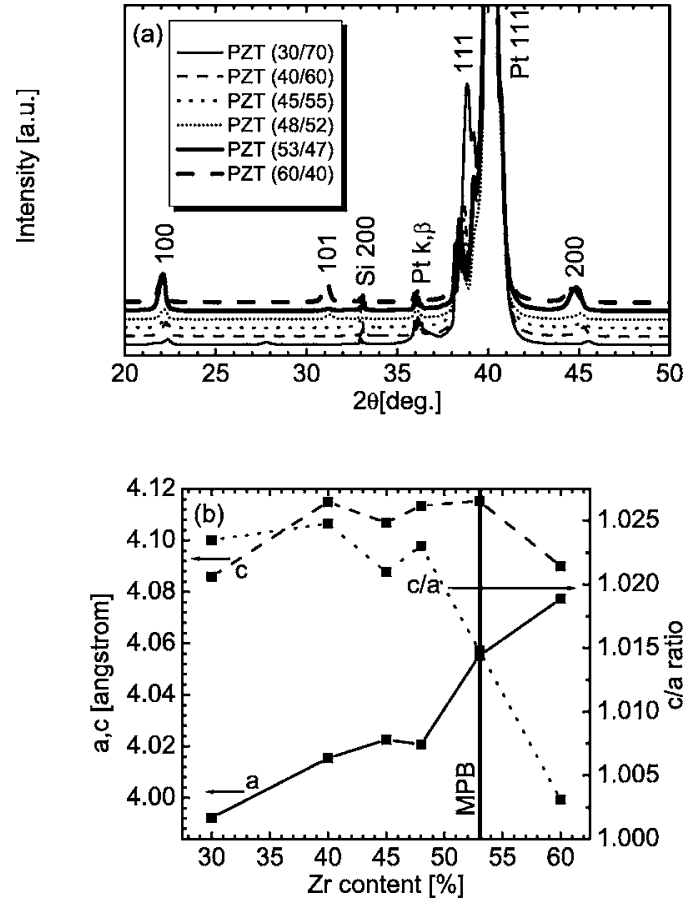

FIG. 1. (a) XRD scans of PZT thin films with increasing Zr content. (b) Calculated lengths of the unit cells in $a$ (line) and $c$ (dashes) axis directions and $c / a$ ratio (tetragonality, dots) vs the $\mathrm{Zr}$ content of the films.

bined with the facts that the (001) peak is nearly absent in all samples and $90^{\circ}$ domain wall motion is inhibited in thin films, the (100) oriented unit cells should not contribute to the electrical and electromechanical behaviors of the samples. Zr rich compositions also exhibit an additional minor (101) oriented phase, but it is unclear whether these unit cells are switchable. Therefore, the behavior of all samples is mainly produced by the (111) oriented unit cells, which is important for further theoretical analysis.

In Fig. 1(b) the calculated lengths of the $a$ and $c$ axes of the unit cells are shown. Additionally, the calculated tetragonality $(c / a$ ratio) is shown in this graph. The $c$ axis length shows an increase from 4.09 to $4.11 \AA$ for tetragonal materials, but stays constant around this value for higher $\mathrm{Zr}$ contents. After passing the morphotropic phase boundary, the length decreases again to $4.09 \AA$. On the other hand, the $a$ axis length increases steadily from 3.99 to $4.02 \AA$ for increasing $\mathrm{Zr}$ content until the composition nears the morphotropic phase boundory (MPB). After that point, the axis length increases rapidly to the end value of $4.08 \AA$. Hence, the $a$ axis length becomes comparable to the value of the $c$ axis length. Consequently, the tetragonality is constant around 1.23 for tetragonal compositions and decreases to nearly 1 on the rhombohedral side. Therefore, the samples are phase pure tetragonal or rhombohedral on either side of the MPB. Only the sample with $53 \% \mathrm{Zr}$ content cannot be sorted into one of the regimes. Hence, both phases or some kind of mixed phase can be present in this sample.

The volume of the unit cells increases with rising $\mathrm{Zr}$ content which can be attributed to the higher ion radius of the $\mathrm{Zr}$ ions. Ti ions only show a radius of $61 \mathrm{pm}$ compared to $87 \mathrm{pm}$ of $\mathrm{Zr}$ ions. Hence, the more $\mathrm{Zr}$ ions are integrated in the ceramic, the higher the average cross section of the unit 


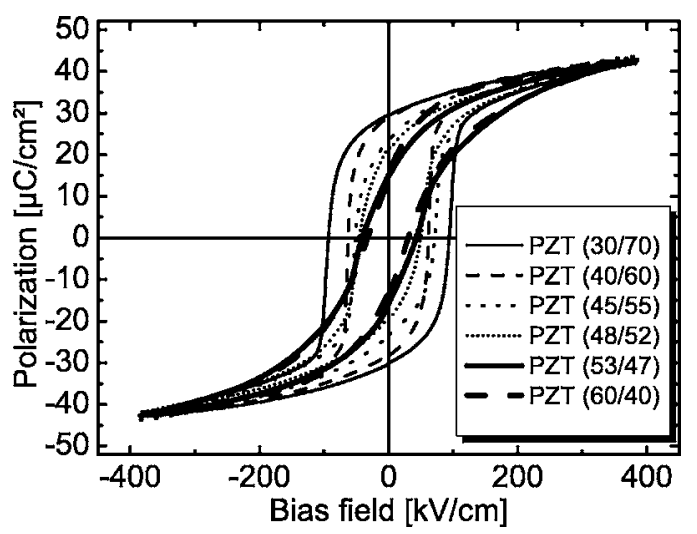

FIG. 2. Electrical large-signal polarization $P$ of PZT thin films with increasing $\mathrm{Zr}$ content $\left(f_{\text {bias }}=1 \mathrm{~Hz}\right)$.

cell becomes. Since there is no measurement technique to measure single unit cells, it cannot be said whether all unit cells become bigger, or only the unit cells containing $\mathrm{Zr}$ ions show an increased size. Theoretically, an increased $\mathrm{Zr}$ containing unit cell should also influence its Ti containing neighbors. Additionally, $\mathrm{Zr}$ becomes the ion responsible for the minimal unit cell size, with the oxygen ions arranged around them. After passing the MPB, the preferred unit cell distortion changes from tetragonal to rhombohedral. Unfortunately, only one sample in this regime is available. Therefore, no prediction about the influence of even higher $\mathrm{Zr}$ contents can be made. Again, neighboring unit cells should influence each other. Hence all unit cells show tetragonal distortion, until a critical limit of $\mathrm{Zr}$ containing unit cells is reached and all unit cells are distorted rhombohedral. The latter is only true if the allocation of $\mathrm{Zr}$ containing unit cells is homogenous in the sample.

\section{B. Electrical behavior}

In the following, the results from the structural analysis and theoretical considerations should be kept in mind, when the electrical behavior is investigated. Starting with the large-signal measurements (Fig. 2), it appears that the polarization $P$ reached in saturation is independent of the material composition. The maximum polarization $P_{\max }$ and minimum polarization $P_{\text {min }}$ are the same for all samples. This is interesting, since $\mathrm{Zr}$ rich samples have a lower density of unit cells due to their increased size. However, the size difference is below $10 \%$. Also, any losses due to the lower density can be compensated by a higher spontaneous polarization or increased intrinsic contributions. In the case of rhombohedral samples, the component of the spontaneous polarization in $Z$ direction can be higher due to the different orientation of the domain vectors.

More obvious is the fact that the coercive field $E_{c}$ decreases for higher $\mathrm{Zr}$ contents. This is accompanied by an earlier start and later ending of the switching process, which is also called "soft" switching behavior, in analogy to ferromagnetic materials. For rhombohedral materials, the switching and the saturation regime of the hysteresis curve seem to melt into each other and no clear boundary can be seen between those two. For compositions near the morphotropic

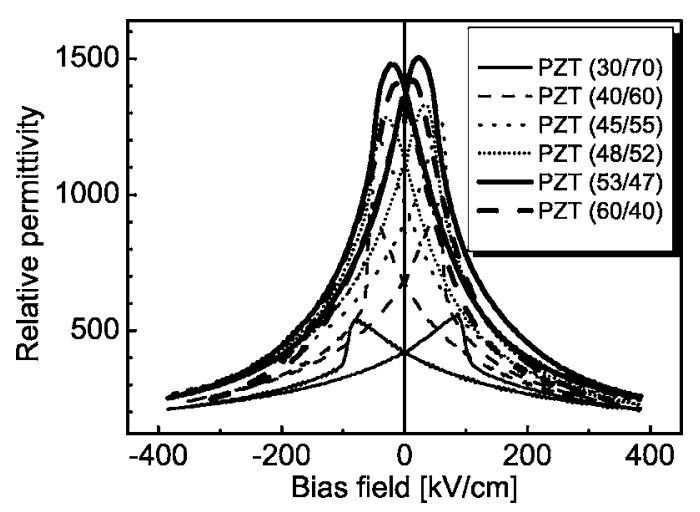

FIG. 3. Electrical small-signal permittivity $\varepsilon_{r}$ of PZT thin films with increasing $\mathrm{Zr}$ content $\left(f_{\text {bias }}=1 \mathrm{~Hz}, f_{\mathrm{ac}}=8 \mathrm{kHz}\right.$, and $\left.V_{\mathrm{ac}}=100 \mathrm{mV}_{\mathrm{rms}}\right)$.

phase boundary, the switching process starts even before opposing fields are applied to the sample which is known as backswitching. With increasing $\mathrm{Zr}$ content, the backswitching starts at higher fields and more of the polarization is lost, before the applied field reached zero again. Hence, stored information is lost in $\mathrm{Zr}$ rich materials, which is a disadvantage, if these materials are considered as memory materials.

As a first suggestion for the reason behind the $\mathrm{Zr}$ influence on the switching behavior, the increased unit cell size could be responsible. Since the $\mathrm{Zr}$ containing unit cells stretch their $\mathrm{Ti}$ containing neighbors, the $\mathrm{Ti}$ containing unit cells become less stable in their equilibrium points. Or, microscopically thinking, in larger unit cells the oxygen square in the center level of the unit cell is wider (see Ref. 21). Therefore, it becomes easier for the center ion to pass through the center of the square, benefiting the switching processes. As a final note, the influence of increasing $\mathrm{Zr}$ content seems to be reduced going from PZT (30/70) to PZT (40/60). Only the coercive field decreases, but the switching process is comparable in its abruptness. Also, the remanent polarization $P_{r}$ in both samples is comparable in these materials.

For further insight, the electrical small-signal behavior (Fig. 3) is investigated next. The main influence of the composition on the small-signal response is an increase of the maximum and remanent of the relative permittivity $\varepsilon_{r}$ for increasing $\mathrm{Zr}$ content. This behavior is true even for very low small-signal amplitudes. Hence, the increased response is not only coming from the changed switching behavior, but is also a result of an increased intrinsic behavior. However, this increase seems to be limited to sample states near the coercive field, since the values become comparable far in saturation. Again, the increased unit cell size could be the reason for the changed material behavior. The increase not only benefits the switching processes, but also improves the mobility of the center ions around their equilibrium states. Even in saturation, the mobility and hence the small-signal response are increased in $\mathrm{Zr}$ rich materials. This is one of the reasons for the measured polarizations in saturation being the same for all samples.

Also, the previously examined influence on the switching behavior is reflected in the small-signal measurements. However, the influence is more difficult to see in these mea- 


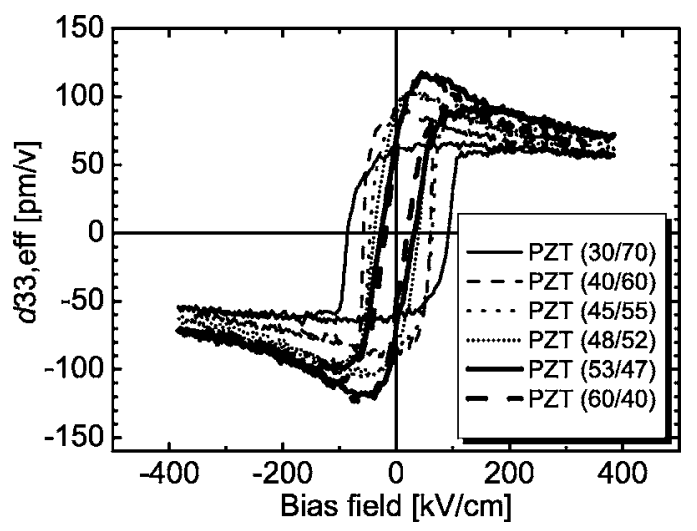

FIG. 4. Electromechanical small-signal coefficient $d_{33 \text {,eff }}$ of PZT thin films with increasing $\mathrm{Zr}$ content $\left(f_{\text {bias }}=1 \mathrm{~Hz}, f_{\mathrm{ac}}=8 \mathrm{kHz}\right.$, and $\left.V_{\mathrm{ac}}=100 \mathrm{mV}_{\mathrm{rms}}\right)$.

surements. The decrease in the coercive field $E_{c}$ is visible as the maximum peaks being closer together, but the remanent values increase faster in relation to the maximum values. The reason for this is that the intrinsic small-signal responses of two unit cells switched into opposing directions are not eliminating each other. The intrinsic current response is always in field direction, as shown in Ref. 22. Therefore, backswitching influences are decreased. On the other hand, the softer switching process can still be seen as less steep drops of the relative permittivity after passing the maximum values. Additionally, the bend in the curve between switching and saturation regimes is smoothed out for $\mathrm{Zr}$ richer materials.

Only the sample with the highest $\mathrm{Zr}$ content of $60 \%$ shows a different influence on its small-signal behavior: The maximum and remanent relative permittivity is decreased compared to the sample with MPB composition. Somehow, the rhombohedral phase decreases the intrinsic response of the material. However, this find should be seen carefully, since only this one sample shows purely rhombohedral unit cells. Also, not all theories found for tetragonally distorted unit cells might be applicable to it.

\section{Electromechanical behavior}

Considering the switching behavior, the $\mathrm{Zr}$ content influence on the coercive field $E_{c}$ is comparable to the one found in the electrical large-signal measurements. It should be noted, however, that the coercive field measured in electromechanical small-signal measurements is not the same, as in the electrical measurements. As was shown in Ref. 23, the $E_{c}$ measured in the electromechnical measurements is slightly higher. For the final comparison, the $E_{c}$ gained in the electrical measurements will be taken into account. The electromechanical small-signal measurements (Fig. 4) show the combined findings from the electrical measurements. Also, the influences reported earlier can be seen more clearly.

In electromechanical small-signal measurements, the switching and saturation regimes can be separated easily. The tilt of the switching process changes with increasing $\mathrm{Zr}$ content. However, the switching regime appears to be smaller in relation to the saturation regimes. This may result from the fact that unit cell switching influences the electro-

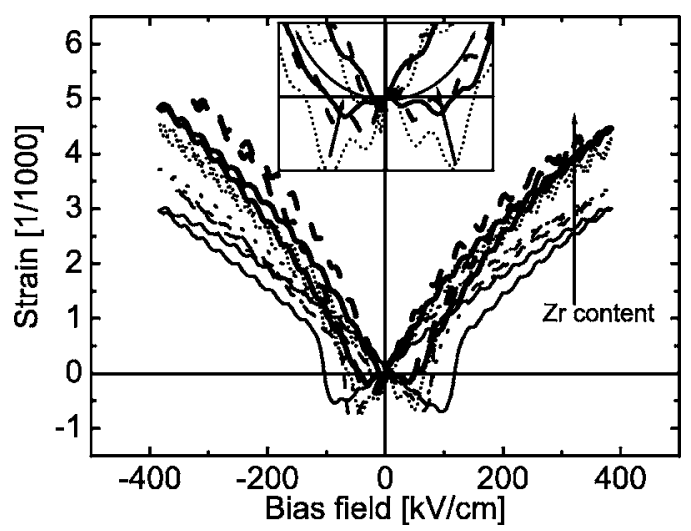

FIG. 5. Electromechanical large-signal strain $S$ of PZT thin films with increasing $\mathrm{Zr}$ content. The thin films measured are PZT (30/70) (line), PZT (40/60) (dashes), PZT (45/55) (dots), PZT (48/52) (short), PZT (53/47) (thick line), and PZT $(60 / 40)$ (thick dashes) (light gray line). ( $f_{\text {bias }}$ $=1000 \mathrm{~Hz}$.)

mechanical behavior only indirectly. ${ }^{21}$ Therefore, its impact might be smaller in sample states, where only a few unit cells are switched against the majority of the unit cells. Again, the $\mathrm{Zr}$ rich samples appear to switch softer than $\mathrm{Ti}$ rich materials. Also, increasing backswitching is visible for compositions with more then $40 \% \mathrm{Zr}$ content, as was reported earlier. Again, PZT (30/70) and PZT (40/60) show a comparable switching behavior, with a decreased $E_{c}$ as the only difference concerning the switching process.

When the values of the effective piezoelectric smallsignal coefficient $d_{33 \text {,eff }}$ are considered, however, PZT (40/60) shows a clear increase in piezoelectric activity. This trend continues until the MPB sample, which shows the highest electromechanical response. As was seen in the electrical small-signal measurements, the rhombohedral PZT $(60 / 40)$ shows a slight decrease in its response. Since both the electrical and the electromechanical small-signal responses are increasing with the $\mathrm{Zr}$ content, it can be concluded that not only the higher mobility of the center ions is responsible for the increased intrinsic behavior. Additionally, the complete unit cells strain more in $\mathrm{Zr}$ rich materials. Hence they appear to be not only increased in size, but also softer in terms of electromechanical stiffness. As a last observation, the electromechanical behavior is increased in the whole saturation regime for $\mathrm{Zr}$ richer samples, if the relative increase is considered. This is somewhat different from the electrical small-signal measurements. There, the increase due to the $\mathrm{Zr}$ influence seemed to decrease with rising bias fields.

The electrical large-signal measurements in Fig. 5 reflect small-signal measurements. Switching becomes softer with increasing $\mathrm{Zr}$ content and the coercive field $E_{c}$ is decreased in such materials. Also, the edge between switching and saturation regimes becomes smoothed out, as is found in the electrical small-signal measurements. More interesting, the minimum negative strain values decrease with rising $\mathrm{Zr}$ content. This is a direct result of the changed switching behavior. In order to reach very low negative strains, the unit cells need to be switched against the direction of the applied electric field. Therefore, only hard switching materials can achieve such values. Softer materials start their switching 
process too early, hence loosing their linearity, if the electromechanical strain is concerned. Hence, $\mathrm{Zr}$ rich materials act less piezoelectric in this regime and more like highly electrostrictive materials (Fig. 5 inlay). This behavior is also indicated by experiments, where the switching behavior is changed by the measurement itself. ${ }^{21}$

On the other hand, the maximum strain increases with rising $\mathrm{Zr}$ content as is expected from the small-signal measurements. The increase is even bigger than in the $d_{33 \text {,eff }}$ measurements, which is a result from the accumulation of the small-signal response in the large-signal behavior. Most interesting is the fact that even the rhombohedral PZT $(60 / 40)$ sample has a larger strain than the MPB PZT $(53 / 47)$ one. This is contradictory to what was expected from the small-signal measurements. The reason for this behavior might be found by the analytical modeling of the samples' large-signal behavior, when all extrinsic influences on the electrical and electromechanical large-signal responses are concerned.

Summarizing the measurement results, $\mathrm{Zr}$ ions increase the unit cell size and force the preferred phase to become rhombohedral after passing the morphotropic phase boundary. The increased unit cell size changes the switching behavior to a more soft switching process and higher backswitching. Also, the mobility of the center ions is increased and the electromechanical stiffness of the unit cells is decreased, both increasing the electrical and electromechanical small-signal responses. The phase change after the MPB reduces this effect somewhat. The latter is not reflected by electromechanical large-signal measurements. Hence the phase change might be accompanied by another effect not clarified so far.

\section{MODELING}

\section{A. Electrical behavior}

Analogous to Ref. 23, the reversible contribution to the polarization $P_{\text {rev }}$ is calculated and depicted in Fig. 6(a). As expected from the measurements of the relative permittivity $\varepsilon_{r}$, the reversible contribution increases with increasing $\mathrm{Zr}$ content to the material composition. Also, the rhombohedral PZT $(60 / 40)$ sample shows decreased $P_{\text {rev }}$ in relation to the MPB sample. In fact, the reversible polarization is as low as the one of PZT $(45 / 55)$. The latter is not expected from the electrical measurements, since only a slight decrease of the electrical small-signal response is measured compared to PZT (53/47). But due to the smaller value of the coercive field $E_{c}$, the rhombohedral sample reaches its peak at lower field values than other samples, which results in the calculated low $P_{\text {rev }}$. The $\mathrm{Zr}$ content influence on the coercive field and the switching behavior is difficult to see in this calculation. But the open area in the center of the curves decreases with rising $\mathrm{Zr}$ content, which reflects the decrease of $E_{c}$.

In Fig. 6(b) on the other hand, the changed switching behavior can be clearly seen again. The irreversible contribution of the polarization $P_{\text {irrev }}$ is highly dependent on the switching behavior, as is shown in Ref. 23. Hence, the calculated $E_{c}$ decreases with rising $\mathrm{Zr}$ content. As with the electromechanical small-signal measurements, these $E_{c}$ values
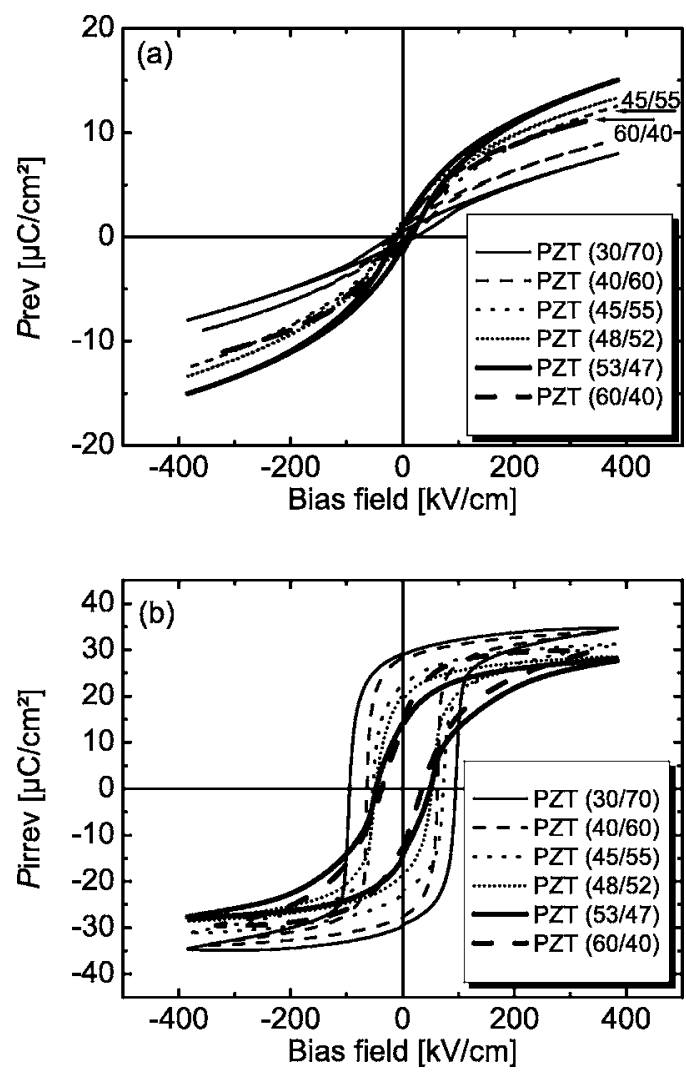

FIG. 6. Calculated electrical large-signal polarization $P$. (a) Reversible $P_{\text {rev }}$ and (b) irreversible $P_{\text {irrev }}$ contributions to the polarization for increasing $\mathrm{Zr}$ content.

are increased in relation to the values gained from the electrical hysteresis measurements. Again, the backswitching starts for $\mathrm{Zr}$ contents larger than $40 \%$ and increases from there, which is reflected by the slope of the curve in the switching regime.

Also interesting, the previously calculated increase of the reversible contribution results directly in a decrease of the irreversible contribution, since the measured polarization $P$ in saturation is the same for all samples. Additionally, the rhombohedral sample of PZT $(60 / 40)$ shows comparable values to the PZT (45/55) sample. Therefore, it can be concluded that together with the phase change in the material, the increased reversible contributions are shifted back to irreversible processes again. An increase in $\mathrm{Zr}$ content and therefore the unit cell size improves the reversible behavior of the material. Upon passing the phase boundary to the rhombohedral regime, this process is reversed, since the internal mechanics of the switching process change. Switching becomes easier again, accompanied by more stable equilibrium positions of the center ion.

This can be explained by the fact that beyond the morphotropic phase boundary, the $\mathrm{Zr}$ containing unit cells become structure defining in the material and the Ti containing unit cells distort the ideal structure. This is exactly the reversed situation as it is in the tetragonal regime before passing the MPB. Hence, it is possible that a further increase of the $\mathrm{Zr}$ ratio might result in even higher irreversible contributions to the polarization and decreased small-signal measure- 

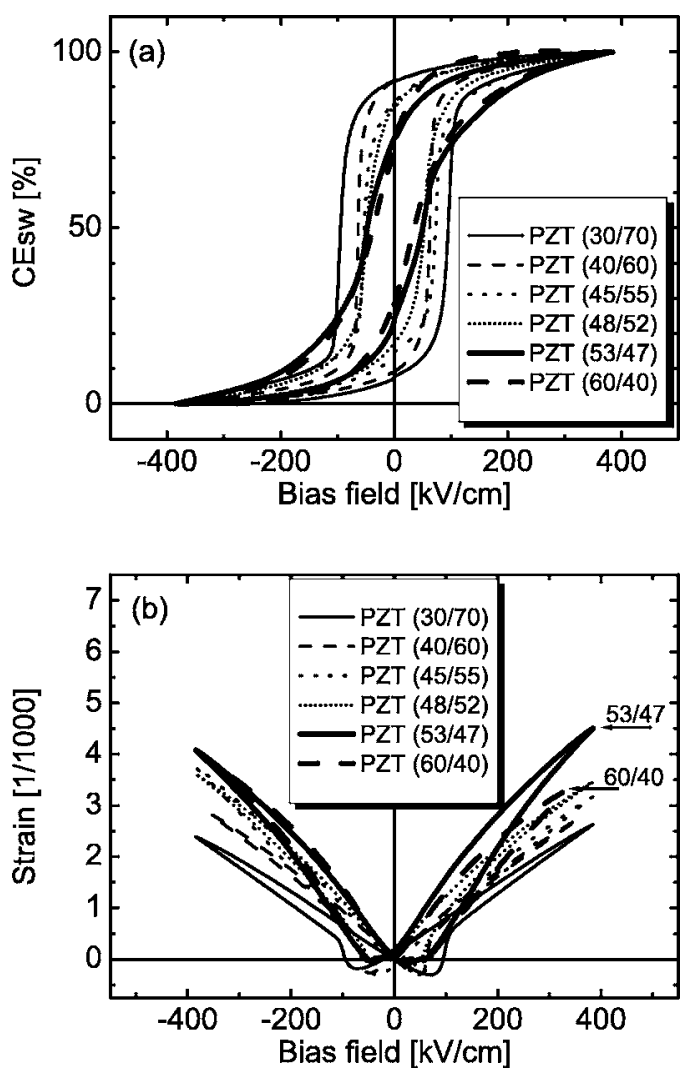

FIG. 7. Calculated (a) percentage of unit cells switched into positive $Z$ direction and (b) electromechanical large-signal strain $S$ for increasing $\mathrm{Zr}$ content.

ments. Of course, the trends seen in the switching behavior should be continuing (even softer switching and further decreased $E_{c}$ ).

\section{B. Electromechanical behavior}

Normalizing the calculated irreversible contribution to the polarization $P_{\text {irrev }}$, by its maximum value, ${ }^{23}$ the ratio of switched unit cells $\mathrm{CE}_{\mathrm{sw}}$ is gained and depicted in Fig. 7(a). The most interesting fact is that $\mathrm{CE}_{\mathrm{SW}}$ of the rhombohedral PZT (60/40) sample is in some regions bigger than the $\mathrm{CE}_{\mathrm{sw}}$ of PZT (30/70), especially in positive saturation. This indicates that the domain configuration under field application in these regimes is more stable for the rhombohedral sample. But once the configuration becomes unstable upon the applied field decreasing, more unit cells switch than in the tetragonal sample.

Also, it takes longer, until the opposite stable configuration is reached for reversed electric fields. On the other hand, reaching the opposite stable configuration takes even longer for PZT (53/47). Therefore, material compositions with $\mathrm{Zr}$ contents larger than $60 \%$ might even show more stable configurations in saturation. It should not be forgotten that the irreversible contribution to the polarization is still lower in rhombohedral materials. The latter is resulting from the softer switching process and the increased reversible contributions. In summary, the domain configuration in saturation is more stable for samples further away from the MPB.

In a final calculation, the electromechanical large signal strain was calculated according to the model developed in
Ref. 23 and is shown in Fig. 7(b). Though the model has certain drawbacks like the used approximation, the calculated curves look comparable to the measured curves in Fig. 5 . The changes to the switching behavior and the decrease of the coercive field $E_{c}$ are modeled well. The edge between switching and saturation regimes smoothes out for increasing $\mathrm{Zr}$ contents, as it did during the measurement. However, negative strain regimes show some differences between model and reality. The minimum reached strain in the model calculations stays constant for samples in the tetragonal regime and changes to nearly zero for the sample near and beyond the morphotropic phase boundary. During the strain measurements, this behavior is more like a constant change with increasing $\mathrm{Zr}$ ratio. Considering the overall picture, the change from a piezoelectric behavior to a more electrostrictive one for small opposing bias fields is visible in both the calculations and the measurements.

The main difference and most interesting fact is again coming from the result of the rhombohedral sample PZT $(60 / 40)$. As expected from the small-signal measurements, the calculated strain curve is smaller than the calculated curve of PZT (53/47). This is contradictory to the measured strain curves gained during electromechanical large-signal characterization. There, the rhombohedral sample yielded the largest strain response of all samples. To elucidate the reason for this discrepancy, it should be remembered that the model calculations only take into account the intrinsic electromechanical behavior and the indirect extrinsic influences on this behavior. Also, measurement errors can be ruled out, since the result is repeatable for different sample capacitors on the sample. This leaves only one explanation for the high electromechanical strain in conjunction with decreased intrinsic behavior: This sample shows a higher direct contribution to the electromechnical strain than the other samples. Remembering the fact that non- $180^{\circ}$ domain wall motions should have no impact on the strain in (111) oriented materials, ${ }^{7,24}$ it should also be noted that this is only true for tetragonal materials and their $90^{\circ}$-switching processes. In rhombohedral materials, the non $-180^{\circ}$ switching is achieved by switching the spontaneous polarization either $71^{\circ}$ or $109^{\circ}$. Therefore, the extrinsic processes can contribute directly to the electromechanical strain. Also, this finding is an evidence for non$180^{\circ}$ domain wall motions, which should be locked in thin films due to substrate clamping, according to Ref. 25.

To summarize the results from analytical modeling, the results gained during the measurements are reflected. Additionally, the domain configuration in saturation seems to be more stable for compositions further away from the morphotropic phase boundary. Also, thin films with rhombohedral not only show non- $180^{\circ}$ domain wall motion, but also a significant contribution of these motions to the electromechanical large-signal strain $S$. Hence, these materials might be of increased interest in future research.

\section{APPLICATION SUITABILITY}

As a final step, the characteristic values for all measurements and calculations are compared to check how they reflect the individual findings. Also, it is investigated how 

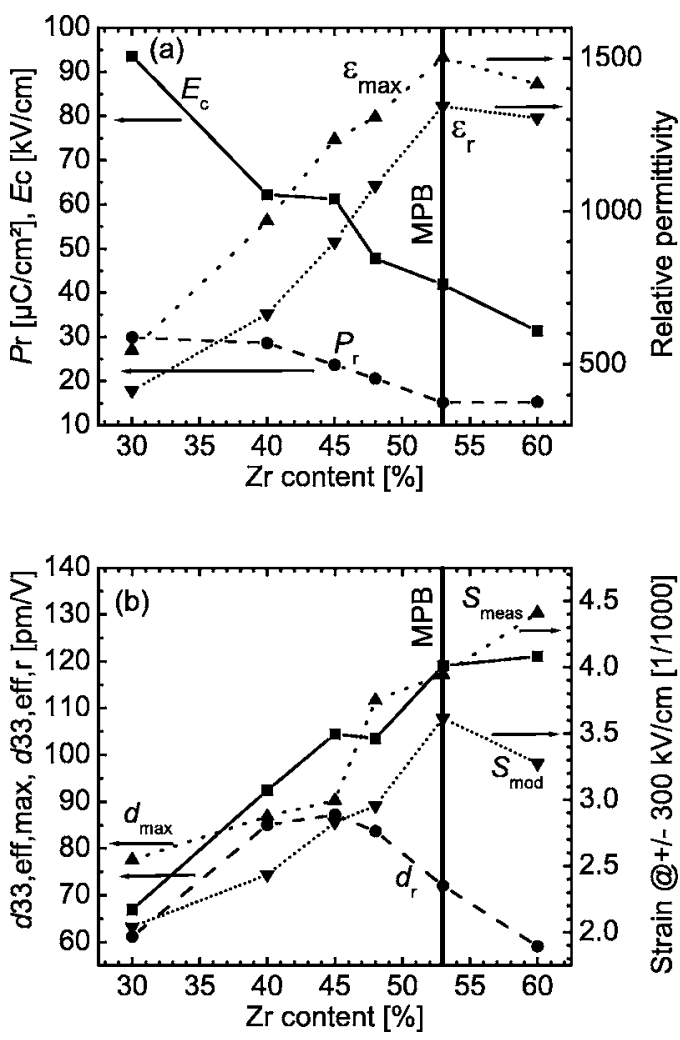

FIG. 8. Characteristic values of the (a) electrical [coercive field $E_{c}$ (line, squares), remanent polarization $P_{r}$ (dashes, circles), peak $\varepsilon_{\max }$ (dots, up triangles), and remanent $\varepsilon_{r}$ (dots, down triangles) permittivity] and (b) electromechanical [peak piezoelectric coefficient $d_{33 \text {,eff,max }}$ (line, squares), remanent piezoelectric coefficient $d_{33, \text { eff r, }}$ (dashes, circles), measured (dots, up triangles), and calculated (short dots, down triangles) strain $S$ at $+l$ $-300 \mathrm{kV} / \mathrm{cm}$ ] behaviors vs the $\mathrm{Zr}$ content.

these values can be utilized to decide about the material suitability for different applications. The four most interesting electrical values: the coercive field $E_{c}$, the remanent polarization $P_{r}$, the peak $\varepsilon_{\max }$, and remanent $\varepsilon_{r}$ permittivity are given in Fig. 8(a).

The changed switching behavior is reflected by decreasing coercive fields $E_{c}$ and remanent polarizations $P_{r}$ for increasing $\mathrm{Zr}$ content. Looking at $E_{c}$ alone gives only limited insight about the switching behavior, since both earlier switching characteristics and starting/increasing backswitching can result in a decrease of $E_{c}$. Additionally, looking at the remanent polarization $P_{r}$ helps us to separate. For PZT $(30 / 70)$ and $(40 / 60), P_{r}$ is nearly constant, showing that the decrease in $E_{c}$ is a result of earlier switching, but not of backswitching, which only starts for higher $\mathrm{Zr}$ contents. Also, $P_{r}$ is the main value to look for, if a suitable material for memory applications is needed. Judging from these results, both PZT (30/70) and PZT (40/60) are equally suitable for memory applications. Since they show different coercive fields, the optimum composition depends on other requirements, e.g., if the material should be more resistant to low voltage spikes [PZT (30/70)] or lower driving voltages are required [PZT (60/40)]. In special cases of extreme low driving voltages, even materials with a higher $\mathrm{Zr}$ content might be selected, though their ability to store information via utilizing $P_{r}$ is reduced.

The relative permittivity $\varepsilon_{r}$ rises with increasing $\mathrm{Zr}$ con- tent until the material phase changes to rhombohedral upon passing the morphotropic phase boundary. Hence, this figure perfectly reflects the results about the Zr contents' impact on the intrinsic processes of the material. The increase in unit cell size and center ion mobility is directly resulting in an increased permittivity. This characteristic value is also the most important for high- $K$ applications, where the material is used to substitute the silicon oxide layers normally utilized to construct capacitors, e.g., in volatile memory chips. Here, the remanent polarization has no importance, since no information is to be stored in the ferroelectric material. On the contrary, a decreased $E_{c}$ and $P_{r}$ might improve the tunability of the device, since the small-signal capacitance changes more for the same change of the bias field. Therefore, $\mathrm{Zr}$ rich materials are the optimal candidates for this area of application. However, until more research is done in the rhombohedral regime, materials on the tetragonal side of the MPB should be utilized. Hence, losses due to the increased extrinsic effects can be avoided.

Microelectromechanical systems (MEMSs) and surface/ bulk acoustic wave (SAW/BAW) devices have one thing in common; they call for large electromechanical behavior in the utilized material usually combined with a high electromechanical coupling factor [Fig. 8(b)]. Depending on the sample geometry, it might be easier to measure the sample strain $S$ or the small-signal coefficient $d_{33 \text {,eff }}$. Also, the characteristic value used for selecting the best material is dependent on the application itself. The maximum and remanent $d_{33 \text {,eff }}$ are good values to reflect the intrinsic material behavior. If the used driving field is small (e.g., SAW/BAW devices), or the direct piezoelectric effect is to be utilized (e.g., sensor application), the remanent $d_{33 \text {,eff }}$ should be used. Suitable compositions are PZT (40/60), PZT (45/55), and PZT (48/52), with PZT (45/55) being the best candidate for small-signal applications.

For large-signal applications, the maximum value of $d_{33 \text {,eff }}$ is of more interest. However, before selecting a material by looking at $d_{33 \text {,eff }}$ alone, the large-signal strain $S$ should be taken into account. This is even more true for rhombohedral materials, where direct extrinsic influences improve the strain response even further, though the small-signal responses stay nearly constant or decrease slightly. Hence, $\mathrm{Zr}$ rich and even rhombohedral [PZT (60/40)] materials should be selected for large-signal applications. However, further research is needed in the rhombohedral region before these materials are used to a greater extent. For instance, the increased extrinsic contributions might lead to an earlier mechanical fatigue of the device, even though ferroelectric fatigue is avoided by unipolar driving.

\section{CONCLUSIONS}

The impact of the material composition on the electrical and electromechanical properties of ferroelectric PZT thin films was measured and modeled. $\mathrm{Zr}$ ions were found to increase the unit cell size and force the preferred phase to become rhombohedral above the MPB. The increased unit cell size changes the switching behavior and increases the intrinsic behavior of the unit cells. The latter is reduced by 
the phase change, which also introduces non- $180^{\circ}$ domain wall motion, improving the large-signal strain. Additionally, the domain configuration in saturation seems to be more stable further away from the MPB. Finally, the most suitable materials were selected for different applications.

${ }^{1}$ N. Setter, Piezoelectric Materials in Devices (Ceramics Laboratory, EPFL Swiss Federal Institute of Technology, Lausanne, 2002).

${ }^{2}$ H. Schaumburg, Keramik (B. G. Teubner, Wiesbaden, 1994).

${ }^{3}$ KFA Juelich, 26. IFF-Ferienkurs (1995).

${ }^{4}$ C. M. Foster, G.-R. Bai, R. Csencsits, J. Vetrone, R. Jammy, L. A. Wills, E. Carr, and J. Amano, J. Appl. Phys. 81, 2349 (1997).

${ }^{5}$ X.-H. Du, J. Zheng, U. Belegundu, and K. Uchino, Appl. Phys. Lett. 72, 2421 (1998).

${ }^{6}$ K. Nagashima and H. Funakubo, Jpn. J. Appl. Phys., Part 1 39, 212 (2000).

${ }^{7}$ S.-Y. Chen and C.-L. Sun, J. Appl. Phys. 90, 2970 (2001).

${ }^{8}$ M. Aratani, T. Oikawa, T. Ozeki, and H. Funakubo, Appl. Phys. Lett. 79, 1000 (2001).

${ }^{9}$ M. Aratani, K. Nagashima, and H. Funakubo, Jpn. J. Appl. Phys., Part 1 40, 4126 (2001).

${ }^{10}$ H. Funakubo, K. Tokita, T. Oikawa, M. Aratani, and K. Saito, J. Appl. Phys. 92, 5448 (2002)

${ }^{11}$ L. M. R. Eakins, B. W. Olson, C. D. Richards, and D. F. Bahr, J. Mater. Res. 18, 2079 (2003).
${ }^{12}$ D.-J. Kim, J.-P. Maria, A. I. Kingon, and S. K. Streiffer, J. Appl. Phys. 93, 5568 (2003).

${ }^{13}$ F. Jona and G. Shirane, Ferroelectric Crystals (Pergamon, Oxford, 1962).

${ }^{14}$ S. H. Oh and H. M. Jang, Phys. Rev. B 63, 132101 (2001).

${ }^{15}$ S. Yokohama, Y. Honda, H. Morioka, T. Oikawa, and H. Funakubo, Appl. Phys. Lett. 83, 2408 (2003).

${ }^{16}$ P. Gerber, A. Roelofs, O. Lohse, C. Kügeler, S. Tiedke, U. Böttger, and R. Waser, Rev. Sci. Instrum. 74, 2613 (2003).

${ }^{17}$ P. Gerber, K. Prume, A. Roelofs, C. Kügeler, U. Böttger, and R. Waser, J. Appl. Phys. 96, 2800 (2004).

${ }^{18} \mathrm{M}$. Lines and A. Glass, Principles and Applications of Ferroelectrics and Related Materials, International Series of Monographs on Solid State Physics (Clarendon, Oxford, 1977).

${ }^{19}$ N. Setter, Piezoelectric Materials in Devices (EPFL Swiss Federal Institute of Technology, Lausanne, 2002).

${ }^{20}$ X. Du, U. Belegundu, and K. Uchino, Jpn. J. Appl. Phys., Part 1 36, 5580 (1997).

${ }^{21}$ P. Gerber, C. Kügeler, U. Böttger, and R. Waser, J. Appl. Phys. 95, 4976 (2004).

${ }^{22}$ P. Gerber, C. Kügeler, U. Ellerkmann, P. Schorn, U. Böttger, and R. Waser, Appl. Phys. Lett. 86, 112908 (2005).

${ }^{23}$ P. Gerber, C. Kügeler, U. Böttger, and R. Waser, J. Appl. Phys. 98, 124101 (2005).

${ }^{24}$ D. Taylor and D. Damjanovic, Appl. Phys. Lett. 76, 1615 (2002).

${ }^{25}$ V. Nagarajan et al., Nature (London) 2, 43 (2002). 